\title{
Correction
}

\section{Correction to: Unconditionally Secure Computation Against Low-Complexity Leakage*}

\author{
Andrej Bogdanov \\ Chinese University of Hong Kong, Shatin, Hong Kong \\ andrejb@gmail.com \\ Yuval Ishai \\ Technion, Haifa, Israel \\ yuvali@cs.technion.ac.il \\ Akshayaram Srinivasan \\ Tata Institute of Fundamental Research, Mumbai, India \\ akshayaram.srinivasan@tifr.res.in \\ Communicated by Jonathan Katz. \\ Online publication 26 October 2021
}

\section{Correction to: J Cryptol (2021) 34:38 \\ https://doi.org/10.1007/s00145-021-09402-2}

This article was updated to replace an incorrect version.

${ }^{*}$ The original article can be found online at https://doi.org/10.1007/s00145-021-09402-2

(C) International Association for Cryptologic Research 2021 